\title{
Perfil de sono e tempo de estudo em acadêmicos do internato de Medicina
}

\author{
Sleep profile and study time in Medical internship students \\ Perfil de sueño y tiempo de estudio en académicos de la pasantía Médica
}

Recebido: 27/10/2021 | Revisado: 01/11/2021 | Aceito: 03/11/2021 | Publicado: 07/11/2021

\author{
Agnes Suelen Paiz dos Santos \\ ORCID: https://orcid.org/0000-0002-7705-8302 \\ Universidade Comunitária da Região de Chapecó, Brasil \\ E-mail: agnessantos@unochapeco.edu.br \\ Letícia Decezaro Dall'Agnol \\ ORCID: https://orcid.org/0000-0002-3463-9801 \\ Universidade Comunitária da Região de Chapecó, Brasil \\ E-mail: decezaro.leticia@unochapeco.edu.br \\ Junir Antonio Lutinski \\ ORCID: https://orcid.org/0000-0003-0149-5415 \\ Universidade Comunitária da Região de Chapecó, Brasil \\ E-mail: junir@unochapeco.edu.br
}

\begin{abstract}
Resumo
O sono participa de inúmeras funções fisiológicas e é o responsável pela manutenção da saúde física e psicológica. Porém, o que se observa nos acadêmicos de Medicina durante o período de internato, é uma carga horária muito extensa que contempla aulas e estágio, fazendo com que a escolha entre qualidade de sono e o bom desempenho acadêmico seja uma necessidade. Este estudo teve como objetivos caracterizar a qualidade do sono de acadêmicos do internato de Medicina e avaliar o impacto do sono na rotina dos acadêmicos de Medicina do quinto e do sexto ano do curso. Os dados para a realização do trabalho foram coletados por meio de um formulário online aplicado aos estudantes do internato no mês de março de 2021. A análise foi feita por meio da interpretação da coleta de dados dispostos em uma planilha no sistema Microsoft Office Excel 2016 e no software Past 4.05. Participaram do estudo 36 alunos, dentre os quais $69,4 \%$ foram mulheres e 30,6\% são homens. Observou-se, que a quantidade média de horas dormidas pelos estudantes foi de 6,03 (DP $\pm 0,84)$, sendo inferior ao recomendado, e a qualidade do estudo é maior quando os acadêmicos têm mais horas de sono, demostrando assim o impacto do sono no bom desempenho acadêmico, fazendo com que sejam necessárias menos horas de estudo para consolidar o conteúdo de forma efetiva.

Palavras-chave: Estudantes de Medicina; Formação acadêmica; Qualidade do sono.
\end{abstract}

\begin{abstract}
Sleep participates in numerous physiological functions and is responsible for maintaining physical and psychological health. However, what is observed in medical students during the internship period is a very extensive workload that includes classes and internships, making the choice between sleep quality and good academic performance a necessity. This study aimed to characterize the sleep quality of medical internship students and assess the impact of sleep on the routine of medical students in the fifth and sixth year of the Medicine course. Data for the performance of the work were collected through an online form applied to boarding students in March 2021. The analysis was performed through the interpretation of data collection arranged in a spreadsheet in the Microsoft Office Excel 2016 system and in the Past 4.05 software. Thirty-six students participated in the study, among which $69.4 \%$ were women and $30.6 \%$ were men. It was observed that the average amount of hours spent by students was 6.03 (SD \pm 0.84$)$, which is lower than recommended, and the quality of the study is higher when students have more hours of sleep, thus demonstrating the impact of sleep on good academic performance, making fewer hours of study necessary to effectively consolidate content.
\end{abstract}

Keywords: Medical students; Academic education; Sleep quality.

\section{Resumen}

El sueño participa en numerosas funciones fisiológicas y es responsable de mantener la salud física y psicológica. Sin embargo, lo que se observa en los estudiantes de medicina durante el período de prácticas es una carga de trabajo muy extensa que incluye clases y prácticas, por lo que la elección entre la calidad del sueño y un buen rendimiento académico es una necesidad. Este estudio tuvo como objetivo caracterizar la calidad del sueño de los estudiantes en prácticas de medicina y evaluar el impacto del sueño en la rutina de los estudiantes de medicina del quinto y sexto año de la carrera. Los datos para el desempeño del trabajo se recolectaron a través de un formulario en línea aplicado a los estudiantes internos en marzo de 2021. El análisis se realizó a través de la interpretación de la recolección de datos dispuestos en una tabla de cálculo en el sistema Microsoft Office Excel 2016 y en el software Past 4.05. En el estudio 
participaron 36 estudiantes, de los cuales el 69,4\% eran mujeres y el 30,6\% hombres. Se observó que la cantidad promedio de horas dedicadas por los estudiantes fue de 6.03 (DE \pm 0.84 ), la cual es menor a la recomendada, y la calidad del estudio es mayor cuando los estudiantes tienen más horas de sueño, demostrando así el impacto del sueño en la buena salud. rendimiento académico, lo que hace necesarias menos horas de estudio para consolidar el contenido de manera efectiva.

Palabras clave: Estudiantes de Medicina; Formación académica; Calidad de sueño.

\section{Introdução}

O sono é responsável por grande parte da manutenção da saúde, tendo participação em várias funções fisiológicas como a consolidação da memória, aprendizagem, restauração de energia e metabolismo, além de garantir qualidade de vida aos indivíduos (Cardoso et al., 2009). O ciclo sono-vigília, regulado pelo ritmo circadiano, é o que determina a qualidade do sono, permitindo um estado de descanso profundo. Perturbações nesse ciclo são a causa de doenças psiquiátricas, doenças cardiovasculares, deterioração no raciocínio e muitos outros prejuízos à saúde (Cardoso et al., 2009).

Dentre os grupos mais propensos a distúrbios no sono estão os residentes e estudantes de Medicina. A alta carga horária do curso, além das diversas atividades para complemento curricular, contribuem para uma sobrecarga dos alunos que, muitas vezes, precisam escolher entre qualidade do sono ou manter um bom desempenho acadêmico (Silva et al., 2016). A intensidade do curso aumenta ainda mais após os quatro primeiros anos, pois nessa etapa se inicia o Internato Médico, equivalente a um estágio, no qual os alunos vivenciam atendimentos e plantões em hospitais com o objetivo de prepará-los para a profissão (Silva et al., 2016).

Ademais, o desempenho acadêmico é fundamental para uma boa qualificação profissional. Isso também é válido para os estudantes de Medicina. Sendo assim, é essencial que eles passem pela universidade com o máximo de aproveitamento possível. Para isso, é necessário que tenham uma boa qualidade de vida, muito associada com uma boa alimentação e boas noites de sono (Cruz et al., 2021). Estudos recentes mostram como o ciclo sono-vigília é importante para a eficiência da aprendizagem, regulando em quais horários o desempenho é maior. Além disso, esse ciclo tem influência na memória, no humor e no comportamento, afetando de forma direta a qualidade de vida das pessoas (Dutra et al., 2021).

Sendo assim, considerando a irregularidade do sono dos estudantes de Medicina, principalmente no período do Internato Médico, além das interferências que isso provoca na aprendizagem, esta pesquisa objetivou compreender a relação entre sono e estudos em acadêmicos do internato de Medicina, através da caracterização da qualidade do sono desses estudantes, para assim avaliar o impacto do sono nos estudos.

\section{Metodologia}

\subsection{Delineamento do estudo}

Estudo caracterizado como transversal que, segundo Hochman et al. (2005), descreve uma situação ou fenômeno de determinada população, independente do período de tempo. Além disso, tem caráter exploratório que, segundo Gil (2002), atua como um meio para se familiarizar com o problema, sendo desenvolvida por entrevistas com pessoas que tiveram experiência prática com o problema e, por fim, são concluídas com uma análise dos dados coletados. Ademais, o estudo possui uma abordagem quantitativa, pois o foco está nos números e nas porcentagens das respostas.

\subsection{População e amostra}

A população consiste nos estudantes do internato de Medicina, anos 5 e 6 do curso, da região de Chapecó, localizado no oeste de Santa Catarina. O município foi fundado em 25 de agosto de 2017 e conta com uma população estimada para 2021 de 227.587 pessoas, em um território de $624,846 \mathrm{~km}^{2}$ (IBGE, 2010). Suas coordenadas geográficas são: Latitude $27^{\circ} 5^{\prime} 48^{\prime \prime}$ 
Sul, Longitude 52 $2^{\circ}$ 37' 7" Oeste. Conta com um PIB per capita de 44.321, 07 R\$, e com 59 estabelecimentos de saúde SUS (IBGE, 2018). O município conta com diversas instituições de ensino, mas apenas duas ofertam o curso de Medicina a Universidade Comunitária da Região de Chapecó (Unochapecó) e a Universidade Federal da Fronteira Sul (UFFS - campus Chapecó).

A amostra foi composta por um grupo de 36 alunos que concordaram com o termo de consentimento livre e esclarecido e responderam de forma voluntária o questionário fornecido. A composição da amostra aconteceu de forma aleatória por livre adesão ao questionário.

Não houve critério para exclusão, já que todas as respostas foram contabilizadas. Referente ao critério de inclusão, se deu pelo livre aceite em participar do estudo.

\subsection{Coleta de dados}

Para a primeira etapa do projeto foi realizada uma pesquisa bibliográfica, definida por Gil (2002, p.44) como o uso de materiais já elaborados, como livros e artigos, para a criação de novos conteúdos. Assim, houve a consulta de artigos científicos para identificar a importância do sono, com foco em estudantes de medicina, além de entender seus efeitos na qualidade dos estudos.

A segunda etapa da coleta de dados foi realizada através da aplicação do questionário online desenvolvido pelos pesquisadores. O questionário iniciava com a caracterização da amostra, perguntando sobre idade, sexo e período do curso, seguindo então com as variáveis voltadas para os objetivos da pesquisa, onde os participantes responderam sobre horas de aula, horas livres e período em que costumavam estudar. Para finalizar o questionário, os participantes classificaram a qualidade de estudos com mais horas de sono e com menos horas de sono (classificação de 0 a 10, onde 0 representa uma péssima qualidade de estudo e 10 excelente). Juntamente com as perguntas, também houve o envio do Termo de Consentimento Livre Esclarecido, garantindo aos participantes o sigilo dos dados por eles fornecidos, além de esclarecer que as informações obtidas somente serão usadas para fins de pesquisa. O questionário aplicado conteve nove questões variando entre opções de respostas abertas e objetivas. Os participantes tiveram um período entre os dias 10 e 20 de março de 2021 para responder o questionário e após esse tempo ele foi fechado para análise e tabulação dos dados obtidos.

\subsection{Análise e interpretação dos resultados}

As informações foram armazenadas em planilhas do programa Excel (Microsoft Inc., 2016) para facilitar a visualização. O banco de dados foi analisado utilizando-se o software Past 4.05 (Hammer, Harper \& Ryan, 2001) e pelo programa Microsoft Excel (2016), utilizando o teste estatístico de normalidade, comparativos pelo teste de Mann-Whitney e do teste de ANOVA: fator único, buscando identificar diferenças entre grupos e subgrupos.

\section{Resultados}

Participaram da pesquisa 36 estudantes. Dessa amostra, 25 (69,4\%) são mulheres e 11 (30,6\%) são homens, com média de idade de 24,22 anos (DP $\pm 1,87$ ). Dos estudantes participantes, 19 (52,8\%) estavam cursando o quinto e 17 (47,2\%) estavam no sexto ano do curso (Tabela 1). 
Tabela 1 - Perfil demográfico dos estudantes do internato de Medicina participantes da pesquisa, Chapecó-SC, 2021. n: número de participantes.

\begin{tabular}{lcc}
\hline Variáveis & $\mathbf{n}$ & $\%$ \\
\hline Sexo & & \\
\hline Masculino & 11 & 30,56 \\
Feminino & 25 & 69,44 \\
\hline Idade (anos) & & \\
\hline 21 & 4 & 11,11 \\
22 & 3 & 8,33 \\
23 & 5 & 13,89 \\
24 & 7 & 19,44 \\
25 & 7 & 19,44 \\
26 & 8 & 22,22 \\
28 & 2 & 5,56 \\
\hline Período do curso & & \\
\hline $5^{\circ}$ Ano & 19 & 52,78 \\
$6^{\circ}$ Ano & 17 & 47,22 \\
\hline
\end{tabular}

Fonte: Autores (2021).

Quanto ao tempo de horas dormidas diariamente, a média dos participantes foi de 6,03 (DP $\pm 0,84)$ (Tabela 2).

Tabela 2 - Frequência absoluta e percentual de horas dormidas dos estudantes do internato de medicina. Chapecó-SC, 2021.

\begin{tabular}{ccc}
\hline $\begin{array}{c}\text { Horas } \\
\text { dormidas/dia }\end{array}$ & $\begin{array}{c}\text { Frequência de } \\
\text { resposta }\end{array}$ & $\begin{array}{c}\text { Porcentagem das } \\
\text { frequências }\end{array}$ \\
\hline 4,5 & 1 & 2,78 \\
5 & 7 & 19,44 \\
6 & 21 & 58,33 \\
6,5 & 1 & 2,78 \\
7 & 3 & 8,33 \\
8 & 3 & 8,33 \\
\hline
\end{tabular}

Fonte: Autores (2021).

O tempo médio, em horas, que os estudantes passam em estágio ou aula foi de 9,50 (DP $\pm 2,27)$ (Figura 1).

Figura 1 - Frequência de horas em estágio/aula dos estudantes do internato de medicina na região de Chapecó-SC.

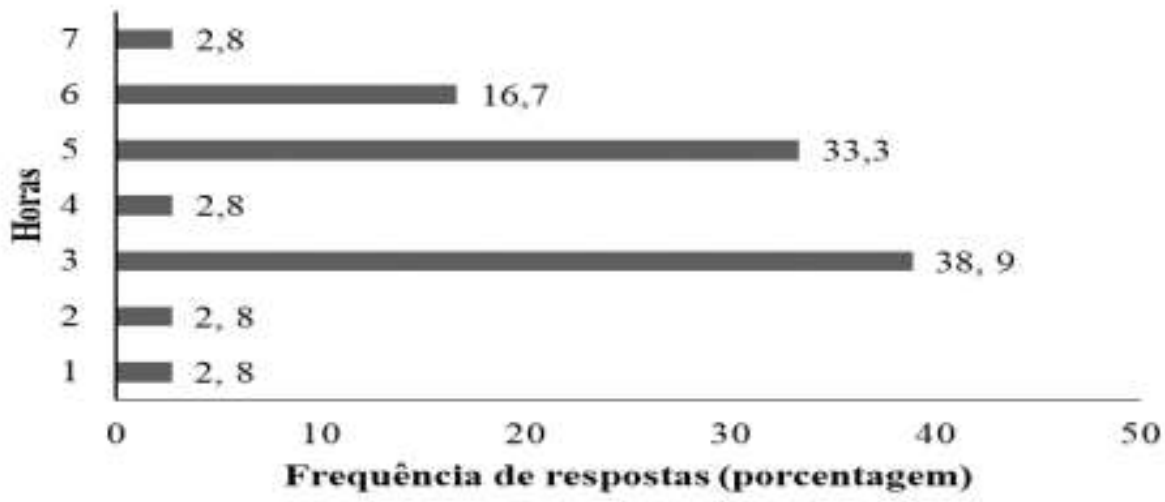

Fonte: Autores (2021).

Quanto à qualidade dos estudos com mais horas de sono, a média dos alunos foi de 8,08 (DP $\pm 1,66)$, enquanto a média 
da qualidade dos estudos com menos horas de sono foi de 3,97 (DP $\pm 2,17)$. Apresentando uma diferença significativa entre as médias ( $\mathrm{p}<0,05$ no teste de Mann-Whitney).

Quanto ao período em que os acadêmicos costumam estudar fora do estágio ou das aulas, 24 (66,7\%) preferem o período noturno, 7 (19,4\%) o período matutino e $5(13,9 \%)$ o período vespertino (Figura 2).

Figura 2 - Período de estudo fora do estágio/aula dos estudantes do internato de Medicina, Chapecó-SC, 2021.

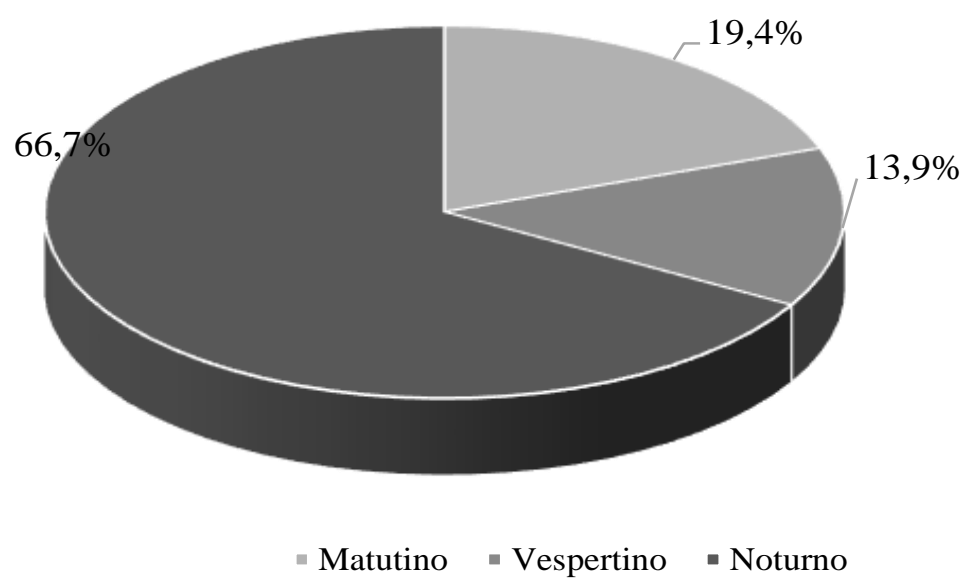

Fonte: Autores (2021).

Dada a elevada carga horária de aula e estágios, a maioria dos estudantes prefere estudar no período noturno (66,7\%). Apesar dessa preferência, o período de estudo não é um fator determinante da qualidade do estudo, mas sim, a quantidade de horas dormidas. Isso foi demonstrado pelo teste de ANOVA, onde, quando foi comparado o "período de estudo" com a "qualidade dos estudos com menos horas de sono" (média=3,97) o resultado não foi significativo ( $\mathrm{F}=3,28 ; \mathrm{p}=0,77)$, assim como a comparação entre "período de estudo" com a "qualidade dos estudos com mais horas de sono" (média=8,08) também não foi significativa $(F=3,28 ; p=0,58)$. Entretanto, quando comparadas às "horas dormidas" (média=6,03; mediana $=6$ ) com "qualidade dos estudos", observou-se resultados significativos ( $\mathrm{p}<0,001)$.

Os estudantes de Medicina costumam sofrer com uma rotina exaustiva, principalmente no período de internato, no qual o $5^{\circ}$ e o $6^{\circ}$ ano não apresentam diferença significativa $(\mathrm{p}=0,50)$ em relação à carga horária diária de estágios e aulas. Consequentemente, ambos possuem uma rotina de sono semelhante, também sem diferença significativa ( $\mathrm{p}=0,06)$.

\section{Discussão}

O perfil da população desse estudo corrobora a literatura (Miranda et al., 2020), sendo composta pela maioria do sexo feminino com a faixa etária média entre 20 e 25 anos.

Foi possível perceber que a alta carga horária do período do internato impacta na quantidade de horas disponíveis para dormir. Com isso, os alunos acabam dormindo menos que as horas diárias recomendadas. Essa realidade também foi identificada por Silva et al. (2016), que constataram uma piora significativa da qualidade do sono dos alunos de Medicina quando entram no período de internato. Os autores ainda mostram que durante os dois últimos anos do curso a maioria dos alunos classifica a qualidade do sono como ruim ou muito ruim. Assim, segundo Oliveira, Hasse \& Teixeira (2021), o internato é um período de grande demanda do estudante, onde, muitas vezes, a falta de administração do tempo e o excesso de cobranças 
e responsabilidades levam a sensação de que nenhum tempo sobra para o estudante descansar.

É possível identificar também, que a carga horária do estudante de Medicina é alta e demanda grande parte do dia dos acadêmicos. Esse achado também foi encontrado no estudo de Silva et al. (2020). No estudo citado, é nítida a insatisfação dos estudantes quanto à carga horária do curso e o curto período disponível para aprender todo o conteúdo necessário. Os autores ainda relataram que o tempo dedicado às atividades acadêmicas dificulta a prática de atividades de lazer e descanso por parte dos estudantes, resultando em uma piora na qualidade de vida.

Como consequência da redução de horas dormidas, os estudantes identificam piora na qualidade dos estudos, indicando que, por estarem mais cansados, sua concentração diminui e seu desempenho acadêmico não é satisfatório. Ademais, baseado no estudo de Leite et al. (2020), as perturbações no sono e, consequentemente, no ciclo sono-vigília, podem desencadear problemas de memória, diminuição do desempenho acadêmico, problemas comportamentais e sonolência diurna. Também foi encontrada uma forte relação entre a má qualidade do sono e o desenvolvimento de depressão durante a faculdade (Nóbrega et al., 2020), mostrando a importância do descanso para a saúde mental e a prevenção de doenças psicológicas.

Ainda com base nos resultados expostos, foi possível perceber a tendência dos acadêmicos do internato de Medicina em escolher o período noturno para estudar. Essa preferência também foi relatada por Miranda et al. (2020), que além disso, identificaram como essa escolha interfere na qualidade de vida dos estudantes, pois esses costumam dormir mais tarde do que a população em geral.

O efeito prejudicial da privação de sono, segundo Purim et al. (2016), está muito associado com o baixo rendimento acadêmico, pois reduz o tempo disponível para atividades de lazer e convívio social, além de dificultar a realização de atividades físicas. Ainda referido pelos autores, isso resulta em estudantes estressados e mais propensos a doenças físicas e mentais. Assim sendo, a dedicação excessiva exigida pelo curso pode acabar afetando de forma negativa a saúde dos estudantes, fazendo-se importante saber administrar as demandas acadêmicas para manter uma boa qualidade de vida, conforme explicado por Dini (2004).

\section{Conclusão}

Concluiu-se que há uma relação entre sono e a qualidade dos estudos dos estudantes de Medicina no período do internato. Notou-se que a carga horária do $5^{\circ}$ e $6^{\circ}$ ano do curso não apresentam diferença significativa, resultando, consequentemente, em uma rotina de sono semelhante. No que tange a qualidade de estudo e as horas dormidas, foi possível entender que se existe uma diminuição nas horas de sono e isso impacta diretamente de forma negativa na qualidade dos estudos dos mesmos.

Dessa maneira, percebe-se a importância do sono para o bem-estar dos acadêmicos e o bom desempenho nos estudos e para que isso seja garantido com maior qualidade é indicado que os alunos do internato de Medicina organizem suas atividades de aulas e estudos para que sejam feitas durante o dia, para que durante a noite possam dormir em horário adequado e melhorem seu desempenho nos estudos e, consequentemente, sua qualidade de vida.

\section{Agradecimentos}

À Universidade Comunitária da Região de Chapecó pelo apoio à pesquisa e a Bolsa de Estudo para Graduação, sendo Bolsa Parcial de 50\% regulamentada pela Lei Federal No 12.101, de 27 de novembro de 2009, e pela LEI Federal No 12.868, de 15 de outubro de 2013.

\section{Referências}


Cardoso, H. C., Bueno, F. C. de C., Mata, J. C. da, Alves, A. P. R., Jochims, I., Filho, I. H. R. V., \& Hanna, M. M. (2009). Avaliação da qualidade do sono em estudantes de Medicina. Rev. bras. educ. med, 33(3), 349-355. https://doi.org/10.1590/S0100-55022009000300005

Cruz, M. C. A., Garcia, T. R., Macedo, R. M., Freitas, Y. J. F. de, Borges, N. M. P., Silva, A. C. S. P. da, Silva, M. L., \& Arruda, J. T. (2021). Influência na qualidade de vida dos estudantes de Medicina relacionadas a má alimentação e sono. Research, Society and Development, 10(2). https://doi.org/10.33448/rsdv10i2.12393

Dini, P. S., \& Batista, N. A. (2004). Graduação e Prática Médica: Expectativas e Concepções de Estudantes de Medicina do $1^{\circ}$ ao $6^{\circ}$ ano. Rev. bras. educ. med, 28(3), 198-203. https://doi.org/10.1590/1981-5271v28.3-026

Dutra, L. da L., Aquino, A. C. N. de, Silva, E. L. da, \& Barros, L. N. (2021). Avaliação do Índice de Qualidade do Sono de Pittsburgh em estudantes de Medicina: Uma revisão integrativa da literatura. Research, Society and Development, 10(8), e52410817530. https://doi.org/10.33448/rsd-v10i8.17530

Gil, A. C. (2002). Como elaborar projetos de pesquisa. Atlas.

Hochman, B., Nahas, F. X., Filho, R. S. de O., \& Ferreira, L. M. (2005). Desenhos de pesquisa. Acta Cir. Bras. 20(2), 2-9. https://doi.org/10.1590/S010286502005000800002

IBGE - Instituto Brasileiro de Geografia e Estatística. Chapecó: panorama. (2010). (2018). https://cidades.ibge.gov.br/brasil/sc/chapeco/panorama.

Leite, B. R., Vieira, T. F. S., Mota, M. de L., Nascimento, E. de C., \& Gomes, I. C. P. (2020). Associação Entre Qualidade de Sono e Ansiedade em Acadêmicos de Medicina. Braz. J. Hea. Rev. 3(3), 6528-6543. https://doi.org/10.34119/bjhrv3n3-200

Miranda, I. M. M., Tavares, H. H. F., Silva, H. R. S. da, Braga, M. S., Santos, R. de O., \& Guerra, H. S. (2020). Qualidade de vida e graduação em medicina. Rev. Bras. Educ. med., 44(3). https://doi.org/10.1590/1981-5271v44.3-20200068

Nóbrega, W. F. S., Oliveira, M. E. C. de, Gomes, K. A. L., Palmeira, J. T., Barbosa, D. V., \& Silva, G. C. B. da. (2020). Depressão na vida acadêmica: quais fatores estão associados? Research, Society and Development, 9(8). https://doi.org/10.33448/rsd-v9i8.5719

Oliveira, S. M. D., Hasse, M., \& Teixeira, F. do B. (2021). Fluxo do esgotamento: interrogando o processo de produção do tempo/cansaço no internato médico. Rev. bras. educ. med. 45(1). https://doi.org/10.1590/1981-5271v45.1-20200243

Purim, K. S. M., Guimarães, A. T. B., Titski, A. C. K., \& Leite, N. (2016). Privação do sono e sonolência excessiva em médicos residentes e estudantes de medicina. Rev. Col. Bras. Cir. 43(6), 438-444. https://doi.org/10.1590/0100-69912016006005

Silva, G. M. da, Ramos, F. A., Bernardes, L. de S., Alves, R. R., \& Fabro, M. A. (2016). Qualidade do sono em estudantes do regime regular e internato médico. Revista Médica da UFPR, 3(1), 19-24. https://doi.org/10.5380/rmu.v3i1.44136

Silva, M. L., Silva, M. L., Silva, A. C. S. P. da, Freitas, Y. J. F. de, Borges, N. M. P., Cruz, M. C. A., Mori, A. S., Macedo, R. M., Garcia, T. R., \& Arruda, J. T. (2020). Condições que interferem na qualidade de vida do estudante de Medicina. Research, Society and Development, 9(11). https://doi.org/10.33448/rsdv9i11.9640 